\title{
Resolving the Connectivity-Throughput Trade-Off in Random Networks
}

\author{
Ralph Tanbourgi, Communications Engineering Lab (CEL), Karlsruhe Institute of Technology (KIT), Germany \\ ralph.tanbourgi@kit.edu
}

\begin{abstract}
The discrepancy between the upper bound on throughput scaling in wireless networks and the throughput scaling in random networks is due to the connectivity-throughput trade-off. In a random network with $\lambda$ nodes, throughput scales by a factor of $\sqrt{\log \lambda}$ worse compared to the upper bound because of the uncertainty in the nodes' location. In this work, nodes know their location and employ power control. The expected throughput-progress is analyzed for a nearest neighbor forwarding strategy, which benefits from power control by reducing spatial contention. By this, the connectivity-throughput trade-off is resolved and the upper bound is achieved.
\end{abstract}

Index Terms-Wireless networks, connectivity, spatial contention, protocol model, nearest neighbor forwarding.

\section{INTRODUCTION}

A lot of effort has been made to analyze the capacity of wireless networks within the last decade. In [1], the authors proved that there exists an upper bound on throughput which scales as ${ }^{1} \Theta(1 / \sqrt{\lambda})$, where $\lambda$ denotes the number of nodes per unit area, i.e., the node density. In [1] the authors also presented a constructive proof for the existence of a global scheduling scheme for which throughput is $\Theta(1 / \sqrt{\lambda \log \lambda})$ in a random network. The proof is based on Voronoi-tessellations, on the assumption of fixed transmission powers, and on routing over straight lines. The gap between this result and the upper bound is due to the additional uncertainty within random networks related to connectivity: for a random network to be asymptotically connected, the transmission range must scale as $\sqrt{\log \lambda / \lambda}$ according to [2], resulting in that the average number of neighboring nodes and hence spatial contention increases with $\log \lambda$. Thus, spatial reuse decreases by a factor of $1 / \log \lambda$ which explains the discrepancy between the throughput result for random networks and the upper bound.

In [3] the authors showed that the upper bound on throughput is feasible also in random networks if the problem of connectivity is tackled differently. They use percolation theory arguments and consider the network in the supercritical regime, where the transmission range of the nodes is chosen to be just above the percolation threshold. In this transition region, a number of paths crossing the network emerge. Packets are then routed along these paths or highways, representing the wireless backbone of the network. On these paths, spatial contention remains constant with increasing $\lambda$. However, as the source as well as the destination node might not be directly connected to a highway, this strategy involves a complicated routing scheme consisting of four phases.

\footnotetext{
${ }^{1}$ In the Landau notation: the term $f(x)=\Theta(g(x))$ denotes the fact that $c_{0}|g(x)| \leq|f(x)| \leq c_{1}|g(x)|$ for $c_{0}, c_{1}<+\infty$ and $x \rightarrow+\infty$.
}

Comparing the two works [1] and [3], one can observe a throughput-connectivity trade-off in random networks: if connectivity is assured, the network is driven in the interference limited regime and throughput is $\sqrt{\log \lambda}$ times lower than the upper bound. On the other hand, the upper bound on throughput is achievable if the network is driven in a connectivity transition region, resulting into a more complex routing scheme.

Fundamental to both models is that a fixed transmission power is assumed. This gives rise to the following question: if we deflect from the assumption of fixed transmission powers, is it possible to ensure connectivity in a random network (for the purpose of a simple routing scheme) and to afford low spatial contention (to achieve the upper bound on throughput) at the same time?

This work represents an extension in the sense that nodes are allowed to employ power control. In fact, we understand power control as an additional degree of freedom that may resolve the connectivity-throughput trade-off and improve network performance. In our network model, the transmission range is chosen to ensure connectivity of the network. However, nodes may reduce their transmission power by transmitting to specific nodes within their range, e.g., to their nearest neighbor. Our approach aims at investigating the average pernode throughput as $\lambda$ increases. We therefore analyze the expected throughput for a given set of parameters, e.g., channel model, interference model, traffic pattern etc. This measure is consistent with prior results in the scaling sense.

\section{A. Related Work and Contribution}

Capacity and throughput scaling laws have been intensively studied in the literature, see for example [1], [3]-[6]. Furthermore, [7] analyzed the scaling behavior of a realistic 802.11 MAC. The throughput-connectivity trade-off was highlighted in [8], [9]. In [8], the impact of different attenuation functions on connectivity and capacity was investigated where it was found that there exist a fundamental trade-off between throughput and connectivity. In [9], the authors proved that the per-node throughput remains constant with increasing $\lambda$, if an arbitrary small fraction of nodes is allowed to be disconnected from the network.

Denote by

$$
\mathcal{T}:=\mathbb{E}\{C Z\}
$$

the expectation of the product of throughput and progress associated with one hop, where $C$ is the per-node throughput or effective (point-to-point) rate in terms of bit per seconds per 
Hertz and $Z$ is the one-hop progress toward the destination in meters. Hence, $\mathcal{T}$ measures how much information has been carried how many meters toward the destination in one second in one hop for one node on average.

Theorem 1: If all nodes employ a nearest neighbor forwarding strategy, we obtain

$$
\mathcal{T}=\Theta\left(\frac{1}{\sqrt{\lambda}}\right)
$$

The remainder of the paper is organized as follows. First, the network model is presented in Section II and the statistical modeling of the spatial contention is explained in Section III. We then give an outline of the solution in Section IV and discuss the implications of these results in Section V. Section VI concludes the paper.

\section{NETWORK MODEL}

\section{A. Network geometry}

A collection of nodes $\left\{X_{i}\right\}$ is assumed to be uniformly and independently distributed on a sphere $A$ with unit area according to a stationary Poisson point process (PPP) with intensity $\lambda$. Let $X_{i}$ denote the $i$-th node as well as its location on $A$. The PPP is conditioned on having $\lambda$ nodes on $A$. The advantage of choosing the sphere rather than some other geometrical topology is that it exhibits a finite surface and avoids unpleasant border effects and allows local observations to be two-dimensional. Initially, each node independently decides whether to originate a packet or to remain silent with probability equal to the network load $p$, where $0<p \leq 1$. All nodes have omnidirectional antennae and obey a maximum transmission power constraint. Furthermore, they are allowed to temporally store an arbitrary number of packets before forwarding. The network is considered at a snapshot of time.

\section{B. Communications model}

As a result of the constraint on maximum transmission power, a node is allowed to communicate directly only with nodes located within its proximity. Here, the motivation for this constraint is that it limits the amount of spatial contention in the network. However, if the range of transmissions is not sufficiently large, connectivity of the network can not be ensured. We therefore set the transmission range equal to the critical radius

$$
r_{c}=\sqrt{2 \frac{\log \lambda-\log \epsilon}{\lambda \gamma}},
$$

i.e., the required radius for which network-wide connectivity is ensured with probability $1-\epsilon$, where $0<\epsilon \ll 1^{2}$. Nodes are further assumed to adjust their transmission power such that the path loss is compensated and a certain signal-to-noise ratio (SNR) is achieved at the receiver. All nodes share a common transmission bandwidth and transmit at the same rate. We further assume that all packets have the same length.

\footnotetext{
${ }^{2}$ The derivation of $r_{c}$ was originally shown in the appendix but was removed due to space limitations.
}

\section{Interference model}

We use the protocol model to characterize interference. The protocol model states that for successful transmission from a transmitter $X_{i}$ to a receiver $X_{j}$ it is required that

$$
\left|X_{k}-X_{j}\right| \geq(1+\Delta)\left|X_{k}-X_{\ell}\right|
$$

holds for all other transmitter-receiver pairs $X_{k}$ and $X_{\ell}$ [6]. The parameter $\Delta$ specifies a guard zone around the receiver which is an increasing function of the spectral efficiency: if transmissions are with high spectral efficiency the required signal-to-interference-plus-noise ratio (SINR) will be high, too. Consequently, interfering neighbors must be farther away so that their emitted power can decay fast enough.

While (3) characterizes interference from the viewpoint of the receiver $X_{j}$, we propose a second inequality that considers interference from the viewpoint of the transmitter: for an interference-free transmission from transmitter $X_{i}$ to receiver $X_{j}$ it is required that

$$
\left|X_{i}-X_{\ell}\right| \geq(1+\Delta)\left|X_{i}-X_{j}\right|
$$

holds for all other receivers $X_{\ell}$.

\section{MAC and Routing scheme}

We assume that transmissions are locally coordinated by the nodes themselves such that each node obtains a time slot in which it can access the medium. This corresponds to a reservation-based TDMA scheduling scheme. Building on the protocol model, a transmission does not experience interference if it is scheduled properly. By properly scheduled we mean that both (3) and (4) hold. Hence, the performance bottleneck is not the interference at the receiver but the split of transmissions in time domain resulting from the spatial contention. We will therefore approximate the effective rate $C$ by the inverse of the number of transmissions that violate either (3) or (4).

We assume that nodes use greedy geographic forwarding (GGF) as routing scheme. In GGF, a node currently holding a packet selects one of its neighbors as relay such that an Euclidean metric is optimized. A node must therefore know its own as well as its neighbors' location and, given it is a source node, it must also know the (approximate) location of the destination node. Generally, GGF may fail due to void areas or node mobility. We will not treat this problem here and assume that GGF does not fail at all. We will also not address the problem of providing location information to nodes. See [10] for details.

We define by $R_{i j}$ the transmission radius, i.e., the distance between transmitting node $X_{i}$ and its corresponding receiver $X_{j}$, as

$$
R_{i j}:=\left|X_{i}-X_{j}\right| .
$$

Furthermore, we define by $Z_{i j}$ the progress, which is the distance between a transmitting node $X_{i}$ and its corresponding receiver $X_{j}$ projected onto a line connecting $X_{i}$ and its corresponding destination node $X_{d}$ (see Fig. 1). We will use the " "nearest with forward progress"، (NFP) strategy as nearest neighbor forwarding scheme. NFP was first introduced in [11] 


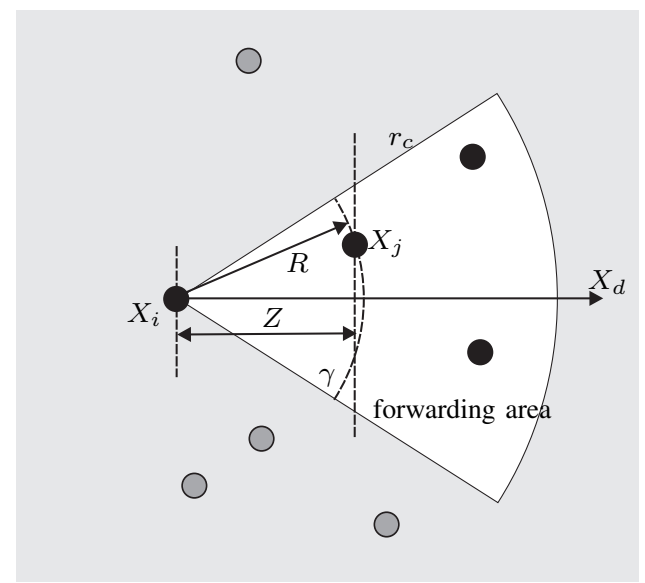

Fig. 1. The NFP scheme: only nodes that are within the forwarding area (white area) are considered as potential relays. Node $X_{i}$ currently holding the packet selects node $X_{j}$ as relay.

and extended by [12] toward a more general forwarding area. In this strategy, node $X_{i}$ selects node $X_{j^{*}}$ as relay if it is in the forwarding area (see Fig. 1) and the transmission radius $R_{i j}$ is minimized. By this, nodes minimize spatial contention. If nodes are distributed according to a homogeneous PPP, the probability density function (PDF) of the distance $R$ is given by the Rayleigh distribution [12]

$$
f_{R}(r)=\lambda \gamma r e^{-\lambda \frac{\gamma}{2} r^{2}}, \quad r \geq 0 .
$$

We condition (6) on the fact that the nearest neighbor is always within distance $r_{c}$ and neglect the normalization term $\frac{1}{1-e^{-\lambda \gamma r_{c}^{2}}}$, i.e.,

$$
f_{R \mid r_{c}}(r)=\frac{f_{R}(r)}{1-e^{-\lambda \gamma r_{c}^{2}}} \simeq f_{R}(r), \quad 0 \leq r \leq r_{c} .
$$

With $1-\epsilon$ denoting the probability of connectivity, it can be shown that the resulting approximation error is less than $\frac{\epsilon / \lambda}{1-\epsilon / \lambda}$ and hence, $\lim _{(\epsilon / \lambda) \rightarrow 0} f_{R \mid r_{c}}\left(r \mid r_{c}\right)=f_{R}(r)$.

We will also use the PDF of $Z$ conditioned on $R$. We can obtain this by simple transformation of random variables, yielding

$$
f_{Z \mid R}(z \mid r)=\frac{2}{\gamma \sqrt{r^{2}-z^{2}}}, \quad r \cos (\gamma / 2) \leq z \leq r .
$$

\section{Statistical Modeling of Medium Access}

\section{A. Modeling from the Receiver's Viewpoint}

Let the random variable $I_{\mathrm{Rx}}$ denote the number of violations of (3) for a typical receiver node $X_{j}$, which is called the reference receiver in the following. Since the PPP is stationary, we can assume without loss of generality that the reference receiver is located in the origin. According to Slivnyak's Theorem, the distribution of a PPP conditioned on having a deterministic point is identical to the distribution of the original PPP [13]. Thus, $I_{\mathrm{Rx}}$ can be written as an independently marked PPP

$$
I_{\mathrm{Rx}}=\sum_{k} K_{k} M_{k}
$$

where $K_{k}$ is a Bernoulli random variable with success probability $p$ and the marks $M_{k} \in\{0,1\}$ indicate that node $X_{k}$ is sufficiently close to create an outage at the reference receiver. The marks $K_{k}$ realize a thinning of the original PPP with thinning factor equal to the network load $p$ (we count only the transmitters). The conditional PDF of the marks $M_{k}$ given position $x_{k}$ follows directly from (3)

$$
f_{M_{k} \mid X_{k}}\left(m_{k} \mid x_{k}\right)=\left\{\begin{array}{l}
\mathbb{P}\left\{(1+\Delta) R_{k \ell} \geq\left|x_{k}\right|\right\}, m=1 \\
\mathbb{P}\left\{(1+\Delta) R_{k \ell}<\left|x_{k}\right|\right\}, m=0,
\end{array}\right.
$$

where the $R_{k \ell}$ is the distance between a transmitter $X_{k}$ and its corresponding receiver $X_{\ell}$. Note that the $M_{k}$ are i.i.d., since every node independently chooses its transmission radius according to the same distribution. The indices $k$ and $\ell$ in (10) can therefore be omitted. The intensity $\Lambda_{\mathrm{Rx}}$ of the Poisson random variable $I_{\mathrm{Rx}}$ is calculated as

$$
\begin{aligned}
\Lambda_{\mathrm{Rx}} & =p \lambda \int_{A} \mathbb{P}\{(1+\Delta) R \geq|x|\} \mathrm{d} x \\
& =p \lambda \int_{A}\left[1-F_{R \mid r_{c}}\left(|x|(1+\Delta)^{-1}\right)\right] \mathrm{d} x,
\end{aligned}
$$

where $F_{R \mid r_{c}}(r)=\mathbb{P}\left\{R \leq r \mid r_{c}\right\}$ is the cumulative density function (CDF) of $R$ conditional on the fact that the nearest neighbor is within $r_{c}$. Note that $1-F_{R \mid r_{c}}\left(|x|(1+\Delta)^{-1}\right)=0$ for $|x|>(1+\Delta) r_{c}$, since transmitters situated outside a disc $d\left(0 ;(1+\Delta) r_{c}\right)$ centered around the receiver (located in the origin) cannot interfere at the receiver due to the fact that the transmission range is limited to $r_{c}$. Thus, (11) can be rewritten as

$$
\Lambda_{\mathrm{Rx}}=p \lambda \int_{d\left(0 ;(1+\Delta) r_{c}\right)}\left[1-F_{R \mid r_{c}}\left(|x|(1+\Delta)^{-1}\right)\right] \mathrm{d} x .
$$

Transforming this into polar coordinates and applying the substitution $\tilde{r}=\frac{r}{1+\Delta}$ yields

$$
\Lambda_{\mathrm{Rx}}=2 p \pi \lambda(1+\Delta)^{2} \int_{0}^{r_{c}} \tilde{r}\left[1-F_{R \mid r_{c}}(\tilde{r})\right] \mathrm{d} \tilde{r} .
$$

The conditional CDF $F_{R \mid r_{c}}(r)$ is obtained from (7) giving

$$
F_{R \mid r_{c}}(r) \simeq 1-e^{-\lambda \frac{\gamma}{2} r^{2}}, \quad 0 \leq r \leq r_{c} .
$$

With (12) and (13) we obtain the intensity of $I_{\mathrm{Rx}}$ for the case when all nodes employ NFP forwarding, according to

$$
\begin{aligned}
\Lambda_{\mathrm{Rx}} & =2 p \pi \lambda(1+\Delta)^{2} \int_{0}^{r_{c}} \tilde{r} e^{-\lambda \frac{\gamma}{2} \tilde{r}^{2}} \mathrm{~d} \tilde{r} \\
& =2 p \frac{\pi}{\gamma}(1+\Delta)^{2}\left(1-\frac{\epsilon}{\lambda}\right) \simeq 2 p \frac{\pi}{\gamma}(1+\Delta)^{2},
\end{aligned}
$$

where the last line follows from the fact that $\frac{\epsilon}{\lambda} \ll 1$. Denoting by $\beta:=p(1+\Delta)^{2}$ the traffic intensity, which is the product of the network load and a function of the spectral efficiency, we can rewrite (14) as

$$
\Lambda_{\mathrm{Rx}}=2 \beta \frac{\pi}{\gamma} .
$$




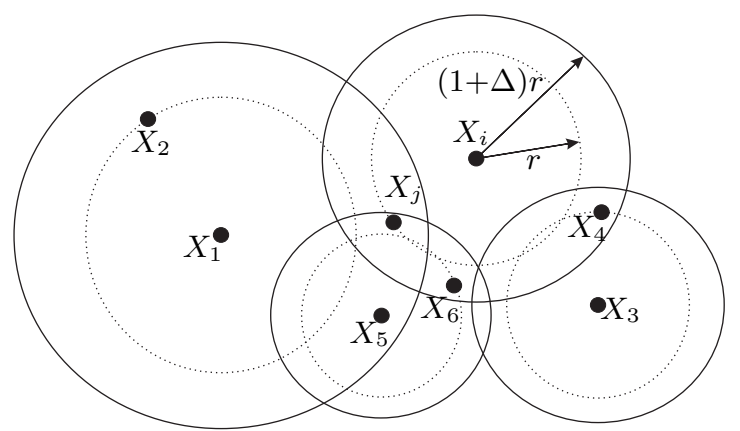

Fig. 2. Illustration of the problem of counting violations twice: the number of violations regarding the reference receiver $X_{j}$ is 2 (pairs $\left\{X_{1}, X_{2}\right\}$ and $\left.\left\{X_{5}, X_{6}\right\}\right)$. Number of violations regarding the reference transmitter $X_{i}$ is 2 (pairs $\left\{X_{3}, X_{4}\right\}$ and $\left\{X_{5}, X_{6}\right\}$ ). Hence, the violation created by the pair $\left\{X_{5}, X_{6}\right\}$ is considered twice.

\section{B. Modeling from the Transmitter's Viewpoint}

We denote by the random variable $I_{\mathrm{Tx}}$ the number of violations of (4) for a typical transmitter node $X_{i}$, which is called the reference transmitter in the following. Here, we assume that the reference transmitter is located in the origin. The number of violations $I_{\mathrm{Tx}}$ can be modeled as a weighted PPP, according to

$$
I_{\mathrm{Tx}}=\sum_{\ell} K_{\ell} \mathbb{P}\left\{R_{i j}(1+\Delta) \geq\left|X_{\ell}\right|\right\},
$$

where $\mathbb{P}\left\{R_{i j}(1+\Delta) \geq\left|X_{\ell}\right|\right\}$ is a function of both the transmission radius $R_{i j}$ between the reference transmitter $X_{i}$ and its corresponding receiver $X_{j}$ and of the distance from reference transmitter $X_{i}$ to a receiving node $X_{\ell}$. The marks $K_{\ell}$ are Bernoulli random variables that realize the thinning of the PPP with thinning factor equal to network load $p$ (we count only the receivers). The term $\mathbb{P}\left\{R_{i j}(1+\Delta) \geq\left|X_{\ell}\right|\right\}$ in (16) can be seen as a weighting of the PPP. The intensity $\Lambda_{\mathrm{Tx} \mid R}$ of the Poisson random variable $I_{\mathrm{Tx}}$ conditioned on the fact that the transmission radius is $R_{i j}=r$ is calculated as

$$
\Lambda_{\mathrm{Tx} \mid R}(r)=p \lambda \int_{A} \mathbb{1}(|x| \leq r(1+\Delta)) \mathrm{d} x .
$$

Since the transmission range $R$ is limited to $r_{c}$, a receiver situated outside a disc $d\left(0 ;(1+\Delta) r_{c}\right)$ centered around the transmitter (located in the origin) cannot be in outage by this transmitter. Hence, the integration range $A$ can be replaced by $d\left(0 ;(1+\Delta) r_{c}\right)$.

$$
\begin{aligned}
\Lambda_{\mathrm{Tx} \mid R}(r) & =p \lambda \int_{d\left(0 ;(1+\Delta) r_{c}\right)} \mathbb{1}(|x| \leq r(1+\Delta)) \mathrm{d} x \\
& =p \lambda \int_{d(0 ;(1+\Delta) r)} \mathrm{d} x=\lambda \pi \beta r^{2} .
\end{aligned}
$$

\section{Optimistic and Pessimistic Modeling}

The number of violations $I$ is certainly made up of the violations $I_{\mathrm{Rx}}$ experienced by the receiver as well as the violations $I_{\mathrm{Tx}}$ caused by the transmitter. However, we can not simply take the sum of $I_{\mathrm{Rx}}$ and $I_{\mathrm{Tx}}$ since this would overrate the true $I$. Fig. 2 illustrates this problem.

To cope with this problem, we suggest an optimistic as well as a pessimistic modeling of $I$ in order to bound the number of violations $I$. In the optimistic case, $I$ is given by $I_{\mathrm{Tx}}$ only. This corresponds to assuming that all violations from the viewpoint of the receiver are already captured by $I_{\mathrm{Tx}}$ which can be seen as a lower bound on $I$. In contrast, a pessimistic modeling for $I$ is given by assuming independence between $I_{\mathrm{Rx}}$ and $I_{\mathrm{Tx}}$ and considering their superposition, which is Poisson with intensity $\Lambda_{\mathrm{Rx}}+\Lambda_{\mathrm{Tx}}$. This can be seen as an upper bound on $I$.

\section{The Solution}

Due to the stationarity of the PPP it is sufficient to consider only one typical transmission, which we will call the reference transmission in the following. The reference transmission represents a typical point-to-point link between the reference transmitter and the corresponding reference receiver. Note that the reference transmitter is not necessarily a source node, but may be a relay as we consider the network in a randomly chosen snapshot. The specified rate at which the packet is transmitted is equal for all nodes. However, if we account for the split in time due to the TDMA MAC, we have to consider the effective rate $C$, which depends on the environment seen by both a transmitter and a receiver through the protocol model, i.e., $C$ depends on the number of violations $I$ associated with a transmission. Hence, for successful transmission, TDMA scheduling implies that

$$
C=\frac{1}{I+1} .
$$

Note that $C$ is a random variable which depends on $I$, where $I$ depends on the transmission radius $R$. To compute $\mathcal{T}$, (19) is further multiplied by the one-hop progress $Z$. The resulting expression is then averaged with respect to the uncertainties $C, Z$ and $R$. Note that $C$ and $Z$ are dependent, since $Z$ and $R$ are. To calculate $\mathcal{T}$ we decompose (1) according to the law of total expectation, yielding

$$
\mathcal{T}=\mathbb{E}\{\mathbb{E}\{Z \mid R\} \mathbb{E}\{C \mid R\}\} .
$$

With (8), $\mathbb{E}\{Z \mid R\}$ is calculated as

$$
\mathbb{E}\{Z \mid R\}=\frac{2 R}{\gamma} \sin (\gamma / 2) .
$$

In case of $\mathbb{E}\{C \mid R\}$, we obtain two expressions, i.e., $\mathbb{E}^{\text {op }}\{C \mid R\}$ and $\mathbb{E}^{\text {pe }}\{C \mid R\}$, according to the optimistic and the pessimistic modeling of $I$. The expected optimistic rate $\mathbb{E}^{\text {op }}\{C \mid R\}$ conditional on $R$ is written as

$$
\begin{aligned}
\mathbb{E}^{\mathrm{op}}\{C \mid R\} & =\mathbb{E}\left\{\frac{1}{I_{\mathrm{Tx}}+1} \mid R\right\} \\
& =\sum_{k=0}^{\infty} \frac{1}{k+1} \frac{\Lambda_{\mathrm{Tx} \mid R}^{k}}{k !} e^{-\Lambda_{\mathrm{T} \times} \mid R}=\frac{1-e^{-\Lambda_{\mathrm{T} \times} \mid R}}{\Lambda_{\mathrm{Tx} \mid R}} .
\end{aligned}
$$

Using (21) and (22), we write the expected optimistic throughput-progress $\mathcal{T}^{\text {op }}$ as

$$
\mathcal{T}^{\text {op }}=\mathbb{E}\left\{\frac{2 R \sin (\gamma / 2)\left(1-e^{-\Lambda_{\mathrm{Tx} \mid R}}\right)}{\gamma \Lambda_{\mathrm{Tx} \mid R}}\right\},
$$


which can be calculated using (7) and (18) as

$$
\begin{array}{r}
\mathcal{T}^{\text {op }}=\frac{2 \sin (\gamma / 2)}{\pi \beta} \int_{0}^{r_{c}}\left(1-e^{-\lambda \pi \beta r^{2}}\right) e^{-\lambda \frac{\gamma}{2} r^{2}} \mathrm{~d} r \\
=\frac{\sqrt{2} \sin (\gamma / 2)}{\sqrt{\lambda \gamma \pi} \beta}[\operatorname{erf}(\sqrt{\log (\lambda / \epsilon)}) \\
\left.-\frac{\operatorname{erf}\left(\sqrt{\log (\lambda / \epsilon)\left(2 \beta \frac{\pi}{\gamma}+1\right)}\right)}{\sqrt{2 \beta \frac{\pi}{\gamma}+1}}\right],
\end{array}
$$

where $\operatorname{erf}(x)=\frac{2}{\sqrt{\pi}} \int_{0}^{x} e^{-t^{2}} \mathrm{~d} t$ denotes the error function. For $\frac{\epsilon}{\lambda} \ll 1$, we have that

$$
\mathcal{T}^{\mathrm{op}} \simeq \frac{\sqrt{2} \sin (\gamma / 2)}{\sqrt{\lambda \gamma \pi} \beta}\left[1-\frac{1}{\sqrt{2 \beta \frac{\pi}{\gamma}+1}}\right],
$$

and we can conclude that

$$
\mathcal{T}^{\mathrm{op}}=\Theta\left(\frac{1}{\sqrt{\lambda}}\right) .
$$

In the case of $\mathcal{T}^{\text {pe }}$ we have to compute $\mathbb{E}^{\text {pe }}\{C \mid R\}$, according to

$$
\begin{aligned}
\mathbb{E}^{\mathrm{pe}}\{C \mid R\} & =\mathbb{E}\left\{\frac{1}{I_{\mathrm{Tx}+\mathrm{Rx}}+1} \mid R\right\} \\
& =\sum_{k=0}^{\infty} \frac{1}{k+1} \frac{\Lambda_{\mathrm{Tx}+\mathrm{Rx} \mid R}^{k}}{k !} e^{-\Lambda_{\mathrm{Tx}+\mathrm{Rx} \mid R}}=\frac{1-e^{-\Lambda_{\mathrm{Tx}+\mathrm{Rx} \mid R}}}{\Lambda_{\mathrm{Tx}+\mathrm{Rx} \mid R}} .
\end{aligned}
$$

Hence, $\mathcal{T}^{\text {pe }}$ can be written as

$$
\mathcal{T}^{\mathrm{pe}}=\mathbb{E}\left\{\frac{2 R \sin (\gamma / 2)\left(1-e^{\left.-\Lambda_{\mathrm{Tx}+\mathrm{Rx} \mid R}\right)}\right.}{\gamma \Lambda_{\mathrm{Tx}+\mathrm{Rx} \mid R}}\right\} .
$$

Using (15), (27) yields

$$
\begin{aligned}
& \mathcal{T}^{\text {pe }}= \frac{2 \sin (\gamma / 2)}{\pi \beta} \int_{0}^{r_{c}} \frac{1-e^{-\lambda \pi \beta\left(r^{2}+\frac{2}{\gamma \lambda}\right)}}{r^{2}+\frac{2}{\gamma \lambda}} r^{2} e^{-\lambda \frac{\gamma}{2} r^{2}} \mathrm{~d} r \\
&= \frac{2 \sin (\gamma / 2)}{\pi \beta}\left[\int_{0}^{r_{c}} \frac{r^{2} e^{-\lambda \frac{\gamma}{2} r^{2}}}{r^{2}+\frac{2}{\gamma \lambda}} \mathrm{d} r\right. \\
&\left.-e^{-2 \frac{\pi}{\gamma} \beta} \int_{0}^{r_{c}} \frac{r^{2} e^{-\lambda \frac{\gamma}{2} r^{2}\left(1+2 \beta \frac{\pi}{\gamma}\right)}}{r^{2}+\frac{2}{\gamma \lambda}} \mathrm{d} r\right] .
\end{aligned}
$$

For the case $\frac{\epsilon}{\lambda} \ll 1$, almost the complete probability mass of $R$ is concentrated within the critical radius $r_{c}$. Thus, the error made by extending the upper integration border to infinity is negligible. With [14], the first integral in (28) then becomes

$$
\begin{aligned}
& \int_{0}^{r_{c}} \frac{r^{2} e^{-\lambda \frac{\gamma}{2} r^{2}}}{r^{2}+\frac{2}{\gamma \lambda}} \mathrm{d} r \simeq \int_{0}^{\infty} \frac{r^{2} e^{-\lambda \frac{\gamma}{2} r^{2}}}{r^{2}+\frac{2}{\gamma \lambda}} \mathrm{d} r \\
& \quad=\sqrt{\frac{\pi}{2 \lambda \gamma}}[1-e \sqrt{\pi}[1-\operatorname{erf}(1)]] \simeq 0.243 \sqrt{\frac{\pi}{2 \lambda \gamma}} .
\end{aligned}
$$

Similarly, the second integral in (28) yields

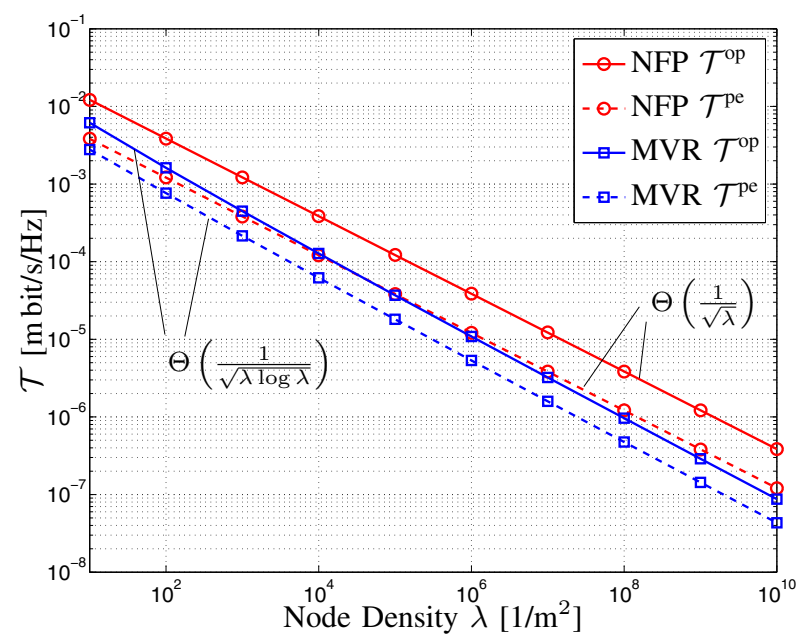

Fig. 3. Expected Throughput-Progress $\mathcal{T}$ for MVR and NFP. Guard zone $\Delta=1$, network load $p=1$, central angle $\gamma=\pi$ and connectivity is ensured with probability $1-\epsilon=0.99$.

$$
\begin{aligned}
& \int_{0}^{r_{c}} \frac{r^{2} e^{-\lambda \frac{\gamma}{2} r^{2}\left(1+2 \beta \frac{\pi}{\gamma}\right)}}{r^{2}+\frac{2}{\gamma \lambda}} \mathrm{d} r \simeq \int_{0}^{\infty} \frac{r^{2} e^{-\lambda \frac{\gamma}{2} r^{2}\left(1+2 \beta \frac{\pi}{\gamma}\right)}}{r^{2}+\frac{2}{\gamma \lambda}} \mathrm{d} r \\
& \quad=\sqrt{\frac{\frac{\pi}{2 \lambda \gamma}}{1+2 \beta \frac{\pi}{\gamma}}}-\frac{\pi e^{1+2 \beta \frac{\pi}{\gamma}}}{\sqrt{2 \lambda \gamma}}\left[1-\operatorname{erf}\left(\sqrt{1+2 \beta \frac{\pi}{\gamma}}\right)\right] .
\end{aligned}
$$

With (29) and (30), we can finally rewrite (28) as

$$
\begin{aligned}
\mathcal{T}^{\mathrm{pe}} \simeq & \frac{\sqrt{2} \sin (\gamma / 2)}{\sqrt{\lambda \gamma \pi} \beta}\left[0.243-\frac{e^{-2 \frac{\pi}{\gamma} \beta}}{\sqrt{1+2 \beta \frac{\pi}{\gamma}}}\right. \\
& \left.+e \sqrt{\pi}\left[1-\operatorname{erf}\left(\sqrt{1+2 \beta \frac{\pi}{\gamma}}\right)\right]\right]=\Theta\left(\frac{1}{\sqrt{\lambda}}\right) .
\end{aligned}
$$

Since $\mathcal{T}^{\text {pe }} \leq \mathcal{T} \leq \mathcal{T}^{\text {op }}$, it follows that $\mathcal{T}=\Theta\left(\frac{1}{\sqrt{\lambda}}\right)$.

\section{IMPLICATIONS}

The outcome of this analysis suggests that the expected pernode throughput in random networks approaches the upper bound in the scaling sense if nodes employ power control in conjunction with NFP. Hence, the throughput-connectivity trade-off can be resolved: by ensuring connectivity with probability $1-\epsilon$, where $0<\epsilon \ll 1$, we can use a simple routing scheme and due to power control, we do not have to drive the network in the self-interference limited regime.

The gain of the NFP strategy can be best illustrated by comparing it to its counterpart, namely the "most forward with variable radius"، (MVR) strategy. While NFP minimizes spatial contention, MVR intends to maximize the progress $Z$ and thus increases spatial contention. If we consider now the case $\frac{\epsilon}{\lambda} \rightarrow 0$ we find that in the MVR strategy $Z \simeq R \simeq r_{c}$, since a node can follow this strategy better and better due to the fact that the average number of potential relays increases with $\log \lambda$. In this case, power control has no effect anymore and the network model becomes similar to the one in [1]. Hence, we expect that employing the MVR strategy results in an expected per-node throughput of $\mathcal{T}=\Theta(1 / \sqrt{\lambda \log \lambda})$. From [11] we can obtain the PDF of $Z$ and the conditional PDF of 


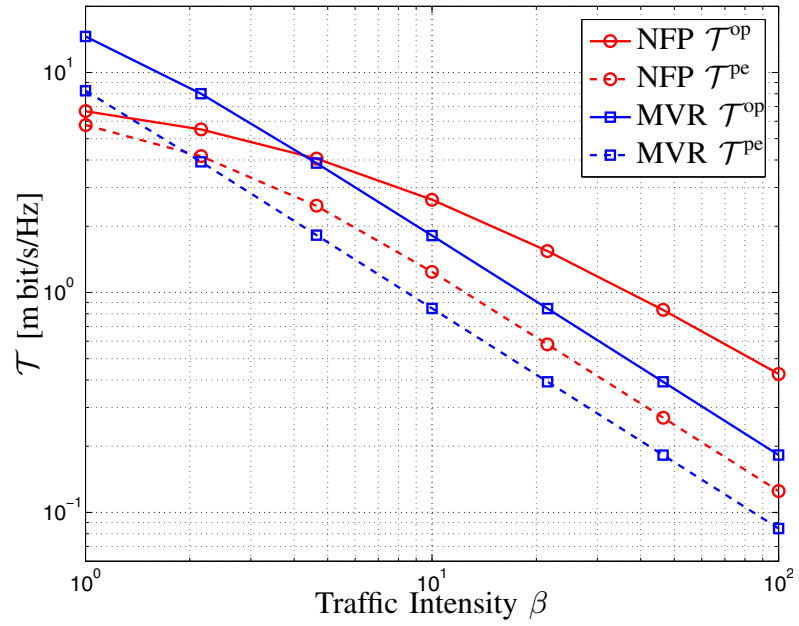

Fig. 4. Expected throughput-progress $\mathcal{T}$ vs. traffic intensity $\beta$ for $\lambda=30$ nodes. The area is set to $|A|=10^{4} \mathrm{~m}^{2}$ and connectivity is ensured with probability $1-\epsilon=0.99$.

$R$ given $Z$, so that we can calculate $\mathcal{T}$ in a similar fashion as in the case of NFP. Fig. 3 shows the scaling behavior of the expected throughput-progress $\mathcal{T}$ for the two forwarding strategies. The results for the MVR strategy were obtained through numerical integration techniques. From Fig. 3 it can be seen that $\mathcal{T}$ is consistent with the results in [1] for the MVR strategy.

An interesting fact of the NFP strategy is the scaling behavior of the expected split of resources a typical node experiences, i.e., the limits $\lim _{\lambda \rightarrow \infty} \mathbb{E}\{I+1\}$ and $\lim _{\lambda \rightarrow \infty} \mathbb{E}\left\{\frac{1}{I+1}\right\}$. These values converge toward constants that depend only on the traffic intensity $\beta$ and on the central angle $\gamma^{3}$. Hence, the amount of spatial contention is independent of the network size for the NFP strategy.

Fig. 4 shows the impact of the traffic intensity $\beta$ on the expected per-node throughput $\mathcal{T}$ for a fixed $\lambda$. It can be seen that for large $\beta$, i.e., high network load and/or large guard zone, the NFP performs better than the MVR strategy. This is because self-interference is the limiting performance factor in the high traffic regime. However, for low $\beta$, spatial contention is comparably small and performance is mainly determined by the one-hop progress $Z$, i.e., $\mathcal{T} \simeq \mathbb{E}\{Z\}$. In this low traffic regime, MVR becomes superior since $Z$ is maximized with this strategy. An optimal forwarding scheme should therefore use an adaptive strategy, taking into account the actual traffic intensity $\beta$ in order to maximize throughput.

\section{CONCLUSION}

We addressed the question if the throughput-connectivity trade-off in random networks can be resolved when nodes are allowed to employ power control. The idea behind this is that nodes can set their transmission ranges such that connectivity in the network is ensured (with probability $1-\epsilon$, where $0<\epsilon \ll 1$ ) while they can reduce their actual transmission power within this range (in order to minimize spatial contention). In this work, power control simply had to compensate for the path loss to ensure a certain received signal strength

\footnotetext{
${ }^{3}$ The values are not derived here due to to space limitations.
}

at the receivers. Furthermore, greedy geographic forwarding was used for modeling the routing scheme. It was found that if nodes use power control in conjunction with a simple nearest neighbor forwarding strategy, i.e., nodes transmit only to their nearest neighbor, the expected per-node throughput is $\Theta(1 / \sqrt{\lambda})$.

Furthermore, we compared the nearest neighbor forwarding strategy NFP to its counterpart strategy MVR, which intends to maximize the one-hop progress. It was found that the latter strategy outperforms the former one for low traffic intensities, i.e., small network loads and small spectral efficiencies. This is because in the low traffic regime, performance is mainly determined by the amount of one-hop progress rather than spatial contention. For high traffic intensities, we found that the NFP strategy performs better because it minimizes spatial contention. We also showed through numerical computation that the expected per-node throughput is $\Theta(1 / \sqrt{\lambda \log \lambda})$ for the MVR strategy which is consistent with the results obtained for random networks in [1].

\section{ACKNOLEDGEMENTS}

The author would like to thank Dr.-Ing. H. Jäkel and Prof. F. K. Jondral for having supervised this thesis.

\section{REFERENCES}

[1] P. Gupta and P. R. Kumar, "The capacity of wireless networks," Information Theory, IEEE Transactions on, vol. 46, no. 2, pp. 388-404, 2000.

[2] P. Gupta and P. Kumar, "Critical power for asymptotic connectivity," in Decision and Control, 1998. Proceedings of the 37th IEEE Conference on, vol. 1, 1998, pp. 1106-1110 vol.1.

[3] M. Franceschetti, O. Dousse, D. N. C. Tse, and P. Thiran, "Closing the gap in the capacity of wireless networks via percolation theory," IEEE Trans. Information Theory, vol. 53, pp. 1009-1018, 2007.

[4] S. R. Kulkarni and P. Viswanath, "A deterministic approach to throughput scaling in wireless networks," IEEE Trans. on Information Theory, vol. 50, pp. 1041-1049, 2004.

[5] A. Jovicic, S. Kulkarni, and P. Viswanath, "Upper bounds to transport capacity of wireless networks," in Decision and Control, 2003. Proceedings. 42nd IEEE Conference on, vol. 3, 9-12 2003, pp. 3136 - 3141.

[6] F. Xue and P. R. Kumar, Scaling Laws for Ad Hoc Wireless Networks: An Information Theoretic Approach, ser. Foundations and Trends in Networking. Now Publishers, vol. 1, no. 2.

[7] J. Li, C. Blake, D. S. J. De Couto, H. I. Lee, and R. Morris, "Capacity of ad hoc wireless networks," in Proceedings of the 7th ACM International Conference on Mobile Computing and Networking, Rome, Italy, July 2001, pp. 61-69.

[8] O. Dousse and P. Thiran, "Connectivity vs capacity in dense ad hoc networks," in INFOCOM 2004. Twenty-third AnnualJoint Conference of the IEEE Computer and Communications Societies, vol. 1, 7-11 2004, p. 486.

[9] O. Dousse, M. Franceschetti, and P. Thiran, "On the throughput scaling of wireless relay networks," Information Theory, IEEE Transactions on, vol. 52, no. 6, pp. 2756 -2761, june 2006.

[10] M. Mauve, J. Widmer, and H. Hartenstein, "A survey on position-based routing in mobile ad-hoc networks," IEEE Network, vol. 15, pp. 30-39, 2001.

[11] T.-C. Hou and V. Li, "Transmission range control in multihop packet radio networks," Communications, IEEE Transactions on, vol. 34, no. 1, pp. 38-44, Jan 1986.

[12] M. Haenggi, "On distances in uniformly random networks," Information Theory, IEEE Transactions on, vol. 51, no. 10, pp. 3584 -3586, 2005.

[13] M. Haenggi, J. Andrews, F. Baccelli, O. Dousse, and M. Franceschetti, "Stochastic geometry and random graphs for the analysis and design of wireless networks," Selected Areas in Communications, IEEE Journal on, vol. 27, no. 7, pp. 1029-1046, September 2009.

[14] I.S.Gradshteyn and I.M.Ryzhik, Table of Integrals, Series, and Products, 6th ed., A. Jeffrey and D. Zwillinger, Eds. Academic Press, 2000. 